\title{
Making quark matter at Brookhaven's new collider
}

Peter Jones, School of Physics and Astronomy, University of Birmingham, Birmingham, UK

$T^{\text {he vast majority of visible matter in the universe consists of }}$ $I$ the protons and neutrons that are found in atomic nuclei. According to our understanding of the structure of matter, protons and neutrons are not fundamental particles in their own right, but are composite particles made of quarks. Quarks are believed to come in six flavours, only the lightest of which, the up and down quarks, are found in protons and neutrons. Particles formed from some of the heavier quarks have only been observed in particle accelerator experiments. Curiously, although a wide variety of particles like the proton and neutron are now known to exist, isolated quarks have never been observed. They appear to exist only in the presence of other quarks.

The reason for this communal existence ultimately has to do with the nature of the strong nuclear force. Quarks are thought to carry one of three so-called colour charges and interact with each other by the exchange of bi-coloured gluons, a process that is described by Quantum-chromodynamics (QCD), a gauge field theory for the strong interaction. What is peculiar about the strong interaction is that the particles that mediate the force, the gluons, are also colour charged. This is not the case for the electromagnetic interaction for example, where the force is mediated by the exchange of photons, which are electrically neutral. This difference is ultimately responsible for the confining property of the strong interaction. Importantly, it is also responsible for determining its short-distance behaviour, which has profound consequences for the structure of matter under extremes of high temperature and density.

As quarks are brought closer together, the force between them decreases dramatically, vanishing as the separation becomes very small. For this reason QCD is said to be an asymptotically free theory. This suggests that quarks may become unbound if the density of quarks could be increased by squeezing a nucleus. In the dense medium formed when the nucleons begin to overlap, another effect comes into play: the interaction between any two quarks in a nucleon becomes screened by their interactions with quarks in neighbouring nucleons. Under these conditions, it becomes meaningless to think of matter as being made up of individual nucleons. The nucleons would have "melted", their constituent quarks now free to roam the extended volume of the compressed nucleus.

The situation described above would mark a significant change in the structure of matter corresponding to a change of phase, rather like the transition from solid to liquid, but in this case from quark confined matter, to a quark-gluon plasma (QGP). This new state of matter has important cosmological and astrophysical implications. It would have been the natural phase of matter until approximately 10 microseconds after the Big Bang, when the temperature of the universe would have been around $2 \times 10^{12}$ kelvin (about 150000 times hotter than the centre of the Sun). It may also exist today in the core of cold but dense stellar objects, such as neutron stars.

\section{Recreating the early universe}

Very little is known about this high temperature and high density phase of matter. Theoretical guidance comes from Monte-Carlo simulations of QCD performed on a discrete space-time lattice. Calculations such as these suggest that the energy density needed to observe the phase transition is around 1 $\mathrm{GeV} / \mathrm{fm}^{3}$, approximately 8 times that of normal nuclear matter. If sufficiently high densities and temperatures could be achieved in the head-on collision of two heavy nuclei at very high energy, it may be possible to recreate this form of matter in the laboratory, albeit over a small volume.

If created in this way, the plasma phase, would be too shortlived to be observed directly. Rather, its existence would have to be inferred from the hundreds of new particles created out of the energy brought into the collision. Theorists have put forward various "signatures", which might be indicative of QGP 


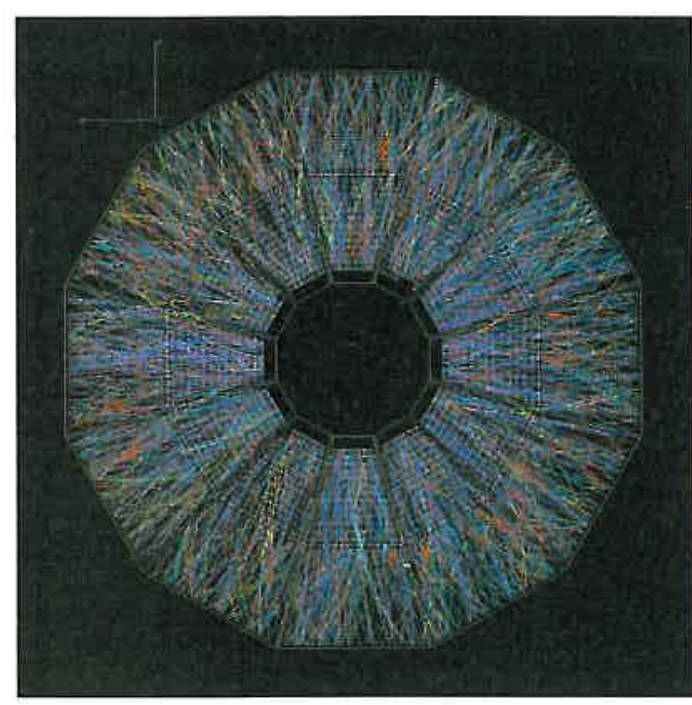

Fig. 1: The head-on collision of two gold nuclei as seen in the STAR experiment. End view of the STAR experiment's main tracking detector, a Time Projection Chamber. The $4 \mathrm{~m}$ high detector is a gas filled cylindrical chamber, which records the trail of ionisation left by charged particles as they pass through the device. The two gold beams enter and leave through the centre of the cylinder and collide approximately mid-way along its $4 \mathrm{~m}$ length. The detector is operated in a magnetic field, which causes positive and negative particles to bend in opposite directions. High momentum particles are bent less than low momentum particles. Each particle trajectory or "track" is colour coded according to its momentum. The detector is designed to measure only those particles emanating from the hot fireball created in the centre of mass of the collision. Approximately 1500 tracks are recorded in a head-on collision of two gold nuclei. formation. In general terms, these are predictions that certain types of particles may be made more easily (or be destroyed) in a QGP, leading to an enhancement (or suppression) of particles in some channels. The experimental challenge is to establish these signatures in the testing environment of high energy heavy ion collisions and to investigate other more conventional explanations for any anomalies that are found.

Attempts to recreate the QGP have been underway at the Brookhaven National Laboratory (BNL) in the United States and at CERN in Switzerland since the mid-1980s. Initially, studies were made colliding rather light projectiles such as oxygen $\left({ }^{16} \mathrm{O}\right)$, silicon $\left({ }^{28} \mathrm{Si}\right)$ and sulphur $\left({ }^{32} \mathrm{~S}\right)$ on a variety of stationary nuclear targets. These first fixed-target experiments demonstrated that it was technically feasible to measure the multiplicity of particles produced in the most violent collisions, but that larger projectiles would be needed to be certain of achieving quark deconfinement over a large enough volume for its effects to be seen. The first truly heavy-ion collisions started in the early $1990 \mathrm{~s}$ when ions of gold $\left({ }^{197} \mathrm{Au}\right)$ and lead $\left({ }^{208} \mathrm{~Pb}\right)$ were accelerated at Brookhaven and CERN respectively. Although similar size ions were used in each case, the highest energy collisions were achieved at CERN where the centre-of-mass energy was more than a factor of three times higher than at Brookhaven. (The centre-of-mass energy is the important quantity here, since it is approximately the amount of energy available for the creation of new particles.)

In February 2000, nearly fifteen years after the start of the heavy-ion programme, CERN announced that compelling evidence had been found for a new phase of matter, which might be the QGP. The evidence, based on a number of key signatures, seemed to indicate that the critical energy density lies between the energies of CERN and Brookhaven. CERN has subsequently embarked on a series of lower energy measurements in an attempt to locate the threshold at which the phase transition occurs. Meanwhile, in the summer of 2000 a new facility was commissioned at Brookhaven that would enable the study of heavy ion collisions at even higher energies.

The Relativistic Heavy Ion Collider (RHIC) is the first facility to be designed primarily to study matter under extremes of high temperature and high density. As a collider of like particles, RHIC consists of two concentric particle accelerators, which the ions tra- verse in opposite directions. The pre-existing accelerator complex at Brookhaven now serves as an injector to the facility, which is capable of producing ions ranging in mass from protons to gold, at centre of mass energies almost 12 times higher than previously possible in fixed-target experiments at CERN. The two beams are brought to collide at 6 points along the 3.8 kilometre circumference of the collider. Four of these interaction regions are currently being used by experiments that have been constructed by international teams of physicists. The four experiments, called STAR, PHENIX, PHOBOS and BRAHMS, have complementary physics goals, but they also share certain common capabilities that provide an important means of comparison between them.

\section{Nuclear stopping power and matter-antimatter symmetry}

During the first experimental run in the summer of 2000, RHIC collided gold ions at a maximum centre-of-mass energy some 7.5 times higher than that previously achieved at CERN. Last year, the beam energy was increased to its design value, corresponding to energies nearly 12 times higher than CERN. At these unprecedented energies, between 4000 and 5000 charged particles are produced in the most violent events, when the nuclei collide head-on (see Figure 1). Under these conditions, the expectation is that the signatures of quark-gluon plasma formation already observed at CERN will be more pronounced. This is because matter will be created at a higher initial temperature. It is also expected that the system of newly created particles will contain almost equal amounts of matter and antimatter, as would have been the case in the Big Bang.

In the early universe, only a very small asymmetry between matter and antimatter, corresponding to an extra baryon for every $10^{10}$ antibaryons, resulted in the universe we see around us. Baryons are the matter particles of the universe. Each baryon contains 3 quarks, whereas an antibaryon contains 3 antiquarks. In heavy-ion collisions, there is an imbalance between matter and antimatter due to the baryons (protons and neutrons) which are brought in with the colliding nuclei. In high energy collisions, nuclei do not completely stop each other, but carry a significant amount of energy away from the interaction. What is left between the two receding nuclei is a hot dense fireball of newly created particles. Since the total number of baryons is conserved in the strong interaction, for every 
new antibaryon a new baryon must also be created, a process known as pair production. It is only the stopping of some of the incoming baryons that gives rise to the imbalance between the number of baryons and antibaryons in the centre-of-mass. If none of the baryons initially contained in the colliding nuclei stop in the collision, the fireball is said to be baryon-free. This means that the fireball contains an equal number of quarks and antiquarks. Such a system will ultimately materialise into an equal number of baryons and antibaryons, as well as other particles, called mesons, which contain both a quark and an antiquark. Together baryons, antibaryons and mesons comprise the family of strongly interacting particles known as hadrons.

One way to quantify the symmetry between matter and antimatter is to measure the ratio of antiprotons to protons, as shown in Figure 2. The value of this ratio at RHIC is found to be around 0.6 , whereas at CERN the ratio was around 0.1 , and much less in the lower energy experiments at Brookhaven. To give these numbers some meaning, the CERN antiproton to proton ratio implies that 9 times more baryons are transported into the centre-

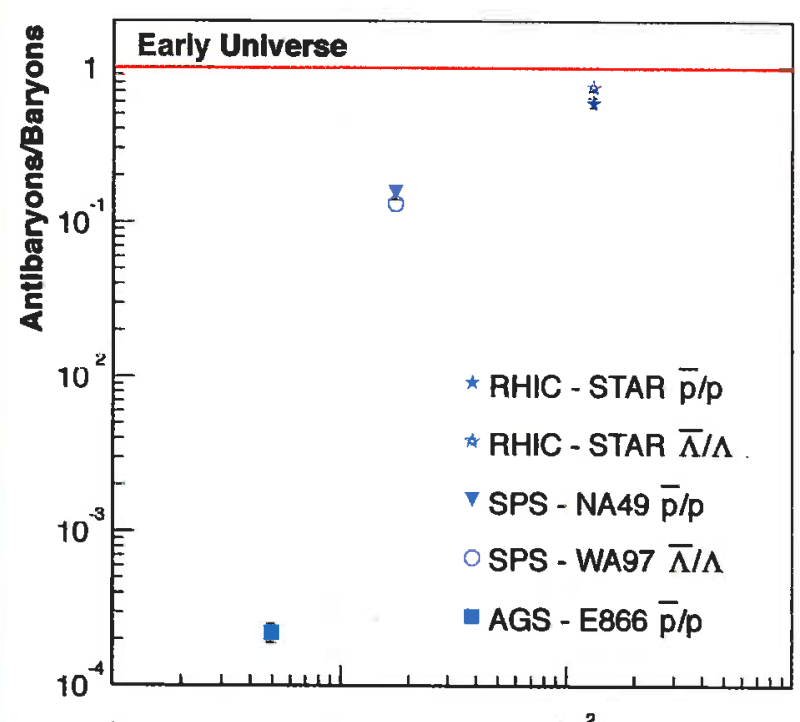

1

10 $10^{2}$

Energy (GeV)

Fig. 2: The asymmetry between matter and antimatter measured by the antibaryon to baryon ratio and plotted as a function of centre-of-mass energy. Almost equal amounts of matter and antimatter were produced in the Big Bang, so that the ratio of antibaryons to baryons would have been very close to unity. Although the number of baryons are conserved in the strong interaction, the fact that nuclei consist of baryons leads to an excess of baryons over antibaryons in heavy ion collisions. This asymmetry depends on the amount of nuclear stopping, which is a function of the centre-of-mass energy of the collisions. Ratios of antiprotons and protons, and antilambdas and lambdas (baryons which also contain a strange quark) are shown, measured at the Brookhaven Alternating Gradient Synchrotron (AGS), the CERN Super Proton Synchrotron (SPS) and at the Brookhaven Relativistic Heavy lon Collider (RHIC). All measurements are made of the fireball created at the centre-of-mass of the colliding system. The ratio of antibaryons to baryons is clearly approaching unity at RHIC, indicating that conditions similar to those that would have prevailed during the early universe are being created. of-mass than are produced, creating a fireball which is rich in baryons. By contrast, the value of the ratio at RHIC implies that 1.5 times more baryons are made by pair production than are stopped in the collision. Although the ratio is clearly approaching unity at RHIC, there is still an excess of matter over antimatter, even at this higher energy. Nevertheless, the conditions created at RHIC are much closer to those of the early universe.

\section{Discovery of jet-quenching?}

Initially, the RHIC experiments will explore how the earlier measurements made at CERN scale with the increased collision energy. They will also look for new reaction features, which may provide a better understanding of the properties of deconfined matter. What is new at RHIC, is that a significant number of particles are thought to originate from the hard scattering of quarks and gluons during the initial stages of the collision. Hard scattering is characterised by a large momentum transfer, leading to a high momentum parton (quark or gluon) travelling transverse to the beam. By the process of confinement, hard-scattered partons fragment into a narrow cone or "jet" of hadrons. At RHIC, jet production is thought to be the main source of particles with a large transverse component of momentum.

A large momentum transfer results from a close encounter between quarks or gluons. Since the strength of the interaction decreases with shorter distances, the cross-section for such processes can be reliably calculated by QCD in a perturbative framework. Furthermore, recent theoretical work has suggested that hard-scattered partons are extremely sensitive to the medium through which they travel. In a dense quark deconfined medium, as in the case of QGP formation, the partons are expected to suffer significant energy loss as they escape the system, leading to a depletion of particles at high transverse momentum. The pattern of energy loss, also known as jet-quenching, is in principle sensitive to the density profile of the surrounding matter, its state of confinement and its overall size. In this way, hard-scattered partons are tomographic probes, rather like annihilation gamma ray photons used in positron imaging, providing information on the distribution of matter formed during the earliest stages of the collision.

The most direct way to search for evidence of jet-quenching is to compare the production rate of high transverse momentum hadrons in heavy-ion collisions with that in proton-proton collisions where there is no surrounding matter to influence the production of jets. Hard scattering is an incoherent process that is expected to scale with the total number of independent nucleonnucleon collisions (also called binary collisions). The number of binary collisions occurring in a gold-gold collision can be calculated from the overlap integral of the two nuclei. This provides a number by which the proton-proton data can be scaled to obtain the expected transverse momentum distribution of hadrons in the absence of jet-quenching or any nuclear effects. This scaling naively assumes that a collision between two nuclei is a 
superposition of independent nucleon-nucleon collisions. By dividing the transverse momentum distribution of hadrons in heavy-ion collisions by the scaled proton-proton distribution, we have a simple way to test for a suppression or enhancement of particles at high transverse momentum. A value of the ratio greater than unity would correspond to an enhancement with respect to a superposition of independent nucleon-nucleon collisions, whereas a value less than unity would correspond to a suppression.

This study has been performed by the STAR and PHENIX collaborations. Figure 3, shows the the transverse momentum distribution of charged particles in gold-gold collisions measured by PHENIX, divided by the scaled proton-proton measurements taken from previously published data. The measured ratio is found everywhere to be lower than the expected value of unity, indicative of a suppression of particles at all transverse momenta. A suppression of particles is to be expected at low transverse momentum where collective effects dominate particle production, which scale with the total number of participating nucleons rather than the number of collisions. Hard processes are only thought to become important above $2 \mathrm{GeV} / \mathrm{c}$, but even here the measured ratio falls approximately a factor of two below the binary-scaling limit.

This observation is made even more striking when compared to CERN data (shown by the dotted lines) where the opposite trend is found. At the lower CERN energy hard scattering does not dominate particle production at large transverse momenta. The enhancement is thought to be due to the multiple scattering of partons in the projectile as they traverse the target nucleus. It is noteworthy that this "normal" nuclear effect works in the opposite direction of jet-quenching, making the observed suppression at RHIC all the more significant.
If these first indications of parton energy loss are confirmed by further studies, researchers will have discovered a promising new tool for studying the properties of the deconfined phase of matter. Over the next few years, the RHIC experiments will undertake a systematic study of jet-production in proton-proton and proton-nucleus collisions as well as in heavy-ion collisions, in order to understand more fully the interplay between nuclear effects and those of parton energy loss. This will be undertaken in parallel with studies of the other signatures of quark-gluon plasma formation already established at CERN, to provide a comprehensive understanding of matter as it would have existed during the first fraction of a second after the Big Bang.

After a successful start-up, RHIC promises to make significant steps toward unravelling the mystery of how quarks and gluons become confined in protons and neutrons. It will remain the world's highest energy heavy-ion collider until around 2008, when CERN aims to complete its next generation particle accelerator, the Large Hadron Collider (LHC).

\section{Further Reading}

New State of Matter created at CERN.

http://press.web.cern.ch/Press/Releases00/List.html

The Relativistic Heavy Ion Collider.

http://www.bnl.gov/rhic

Midrapidity antiproton-to-proton ratio from $\mathrm{Au}+\mathrm{Au}$ collisions at $V_{s_{N N}}=130 \mathrm{GeV}$, C. Adler et al., Phys. Rev. Lett. 86, 4778 (2001)

Effects of jet-quenching on high $p_{T}$ hadron spectra in high energy nuclear collisions, X.N. Wang, Phys. Rev. C58, 2321 (1998)

Suppression of hadrons with large transverse momentum in central $A u+A u$ collisions at ${\sqrt{s_{N N}}}=130 \mathrm{GeV}, \mathrm{K}$. Adcox et al., Phys. Rev. Lett. 88, 022301 (2002)

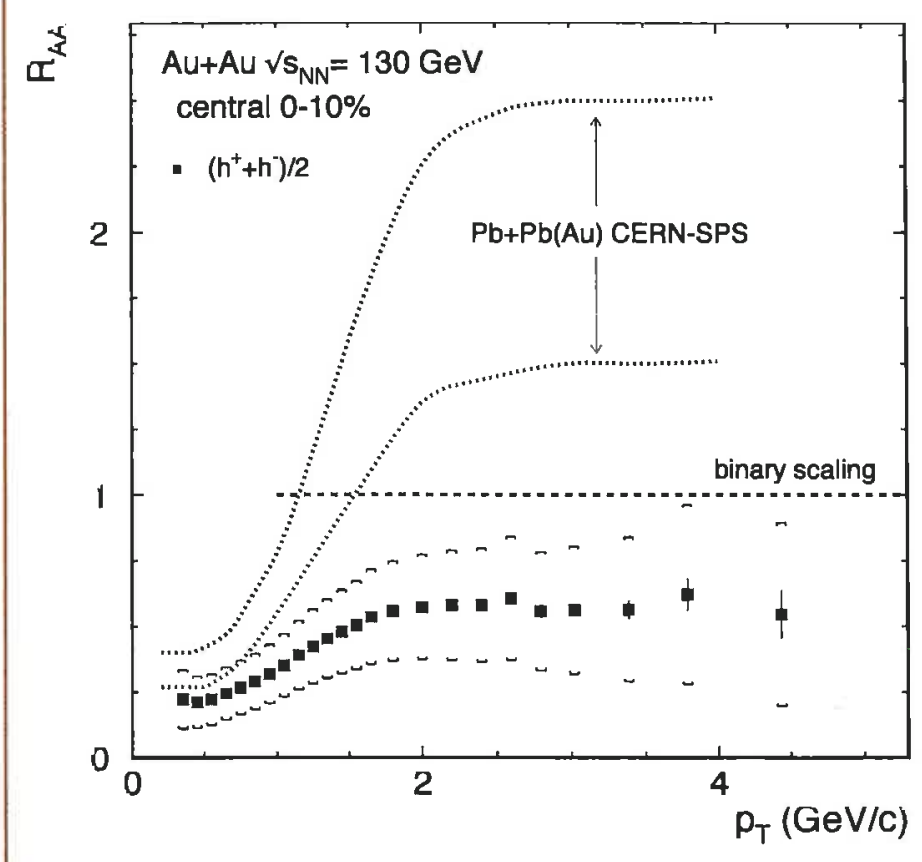

Fig. 3: Evidence for the suppression of high transverse momentum ( $p_{T}$ ) hadrons in $A u+A u$ collisions at RHIC, published by the PHENIX collaboration. The ratio $R_{A A}$ is the distribution of particles produced as a function of transverse momentum (perpendicular to the beam direction) measured in gold-gold and proton-proton collisions. The proton data has been scaled by the number of nucleonnucleon collisions that are expected in the head-on collision of two gold nuclei. This is thought to be the appropriate scaling for hard scattering processes. A value of the ratio less than unity would indicate a suppression of particles relative to a superposition of independent nucleon-nucleon collisions. The data points, which represent the measured ratio for charged hadrons, all fall below unity. The square brackets indicate the overall uncertainty of the measurement. Hard scattering processes are thought to become important only for transverse momenta greater than $2 \mathrm{GeV} / \mathrm{c}$. For comparison the same ratio has been calculated for measurements made at lower energy at CERN, where hard processes are not important. Only the limits of the measurement are shown by the dotted lines. Here, an enhancement is found at high transverse momentum, making the suppression at the higher RHIC energy even more striking. 